\title{
Voltage-Gated Calcium Channel Antibody- Induced Oropharyngeal Dysphagia Presenting as a Paraneoplastic Neurological Complication in Breast Cancer
}

\author{
Razwana Khanam ${ }^{1}$, Ibrahim S. Fanous ${ }^{1}$, Eman N. Fadhel ${ }^{2}$, Tara Hyder $^{3}$, Adam Brufsky ${ }^{4}$ \\ 1. Internal Medicine, University of Pittsburgh Medical Center, McKeesport, USA 2. Family Medicine, University of \\ Pittsburgh Medical Center, McKeesport, USA 3. University of Pittsburgh Physicians, University of Pittsburgh Medical \\ Center, Pittsburgh, USA 4. Hematology/Oncology, Magee Women's Hospital, University of Pittsburgh Medical Center, \\ Pittsburgh, USA
}

Corresponding author: Razwana Khanam, razwanakhanam19@gmail.com

\begin{abstract}
Paraneoplastic neurologic syndromes (PNS) are a group of disorders characterized by an autoimmune response against the nervous system due to cross-reactivity between malignant and normal neural tissue. The most commonly associated malignancies include small cell lung cancer, ovarian cancer, breast cancer, and lymphoma. Multiple PNS have been reported including paraneoplastic cerebellar degeneration, retinopathy, sensorimotor peripheral neuropathy, encephalopathy, opsoclonus-myoclonus syndrome, and stiff-person syndrome.
\end{abstract}

We report a case of a 67-year-old woman with breast cancer who presented with a history of progressive oropharyngeal dysphagia as a paraneoplastic neurologic complication. She was diagnosed with invasive ductal carcinoma, nuclear grade 3 with moderate peritumoral lymphoid infiltrate. Hormone receptors were weakly positive for estrogen receptor (ER) (H score 15), weakly positive for progesterone receptor (PR) (H score 30), and negative for human epidermal growth factor receptor 2 (HER-2/NEU). The patient underwent a localized segmental mastectomy but declined any further adjuvant treatment. Three years after being diagnosed with invasive ductal carcinoma of the breast, she developed progressive oropharyngeal dysphagia that warranted percutaneous endoscopic gastrostomy (PEG) tube placement. Testing for onconeural antibodies was positive for voltage-gated calcium channel antibody. An extensive workup was negative for any alternative etiology that would explain her neurological symptoms. The patient declined further treatment and eventually succumbed to her illness.

Review began 02/22/2021 Review ended 03/01/2021 Published 03/03/2021

\section{() Copyright 2021}

Khanam et al. This is an open access article distributed under the terms of the Creative Commons Attribution License CC-BY 4.0., which permits unrestricted use, distribution, and reproduction in any medium, provided the original author and source are credited.
Categories: Neurology, Oncology

Keywords: invasive ductal cell carcinoma, paraneoplastic syndromes, oropharyngeal dysphagia, voltage-gated calcium channels

\section{Introduction}

Paraneoplastic neurologic syndromes (PNS) are uncommon in solid tumors. They are less frequently seen in association with breast cancer compared to other cancer types such as lung cancer or head and neck cancers. It is unclear whether certain patients' subsets, receptor typing, or histological patterns of breast cancer predispose them to develop PNS [1].

In 2004, an international panel of neurologists established a set of guidelines that would assist in the diagnosis and classification of PNS into two categories: "definite" or "possible" [2]. The diagnostic criteria for "definite" include a "classical" neurological syndrome with onconeural antibodies or cancer that occurs within five years of the diagnosis of neurological symptoms. The term "classical syndrome" applies to those neurological syndromes that have a strong association with cancer. Some of these syndromes include encephalomyelitis, limbic encephalitis, subacute cerebellar degeneration, opsoclonus-myoclonus, LambertEaton myasthenic syndrome, and dermatomyositis. Furthermore, a diagnosis of PNS requires ruling out other potential causes for the neurological symptoms.

Paraneoplastic cerebellar degeneration (PCD) is one of the most common subtypes of PNS affecting the central nervous system. It has been reported in approximately one percent of cancers and has been most commonly associated with small cell lung cancer (SCLC) [3]. Patients with PCD can have a wide range of clinical presentations including truncal ataxia, nystagmus, vertigo, dysarthria, dysphagia, and diplopia. Cognitive disorders and psychiatric symptoms have also been observed [4]. The clinical manifestations are usually bilateral with an asymmetrical distribution [5]. The patient can also present with stroke-like symptoms, tremors, and disorders of speech $[6,7]$. An integral part of diagnosing PCD requires the exclusion of other possible etiologies such as brain metastases, infections, metabolic derangements, vascular, or drug toxicities. 


\section{Cureus}

Historically, PCD has been associated with multiple onconeural antibodies including anti-Yo, anti-Hu, anti$\mathrm{Tr}$, anti-Ri, anti-mGluR1, and anti-voltage-gated calcium channel (anti-VGCC) antibodies. The anti-Yo antibody has been the most commonly reported antibody in association with PCD [5]. VGCC antibodies have been reported in association with Lambert-Eaton syndrome. VGCC antibodies have been reported less frequently in paraneoplastic syndromes. Most of these cases have been in association with SCLC [8]. Approximately $40 \%$ of patients with subacute onset cerebellar degeneration, usually with SCLC, are also positive for the VGCC antibody [3,9-13].

Testing for onconeural antibodies can be done on serum or cerebrospinal fluid (CSF). In one study, 62 cases of PCD were identified and fully characterized between 1966 and 1990. The anti-Yo antibody was detected in the analysis of peripheral blood and CSF samples. This meant that patients did not have to undergo invasive CSF testing in order to make the diagnosis of PCD [14]. Here, we present a case of VGCC antibody-mediated oropharyngeal dysphagia presenting as a paraneoplastic neurological complication in association with invasive ductal carcinoma of the breast.

\section{Case Presentation}

The patient is a 67-year-old woman who was found to have a mass in her left breast on routine mammography. Ultrasonography revealed a $0.6 \times 0.4 \times 0.6 \mathrm{~cm}$ mass (Figure 1 ). A biopsy revealed invasive ductal carcinoma, nuclear grade 3 with moderate peritumoral lymphoid infiltrate. The tumor was weakly positive for estrogen receptor (ER) (H score 15), weakly positive for progesterone receptor (PR) (H score 30), and human epidermal growth factor receptor 2 (HER2)-negative. The tumor proliferation index Ki-67 was $70 \%$.

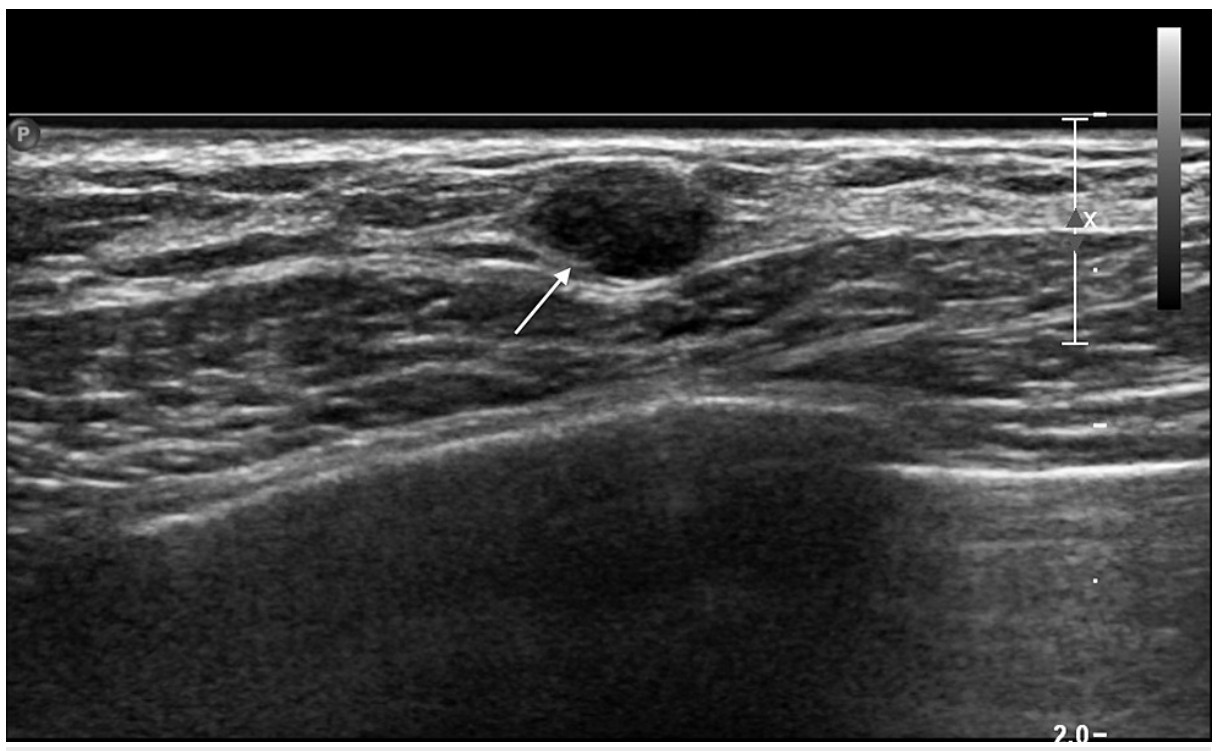

FIGURE 1: Oval hypoechoic circumscribed mass measuring $0.6 \times 0.4 \times$ $0.6 \mathrm{~cm}$ is identified in the 01:00 region (arrow) $7 \mathrm{~cm}$ from the nipple

The patient had a past medical history significant for HIV that was well controlled on zidovudine, lamivudine, and efavirenz. She also had ischemic cardiomyopathy with a reduced ejection fraction of $20 \%$, dyslipidemia, and essential hypertension. Although she had a positive history of breast cancer, she declined BRCA screening. She subsequently underwent left-sided localized segmental mastectomy with sentinel node biopsy. Surgical margins were negative, and four sentinel lymph nodes were negative for tumor as well. She was pathologically staged as pT1bN0. The patient declined any further adjuvant therapy. She continued with an annual surveillance mammogram to detect any evidence of recurrence.

Three years later, the patient started complaining of dysphagia to both liquids and solids with no reported odynophagia. A modified barium swallow demonstrated findings consistent with severe oropharyngeal dysphagia. The patient's symptoms led to malnutrition and failure to thrive and a decision was made to place a percutaneous endoscopic gastrostomy (PEG) tube for nutrition.

A workup was done to identify the cause of her oropharyngeal dysphagia. A flexible laryngoscopy demonstrated normal vocal cords with no anatomical abnormalities to explain her symptoms. The acetylcholine receptor antibodies (AchR-Ab), including binding, blocking, and modulating antibodies, were negative, which ruled out myasthenia gravis. Brain MRI was able to rule out multiple sclerosis, progressive multifocal leukoencephalopathy, and brain metastasis. CSF analysis showed oligoclonal bands that were 
deemed non-specific at that time. Investigations for potential infectious causes were negative for syphilis and Lyme disease. The patient was eventually discharged without determination of the underlying etiology for the oropharyngeal dysphagia.

The patient was readmitted a month later to the intensive care unit for management of acute respiratory failure secondary to pneumonia and sepsis. A CT chest, abdomen and pelvis was negative for any metastatic lesion. During that admission, testing for serum onconeural antibodies was positive for VGCC antibodies $(>30 \mathrm{pmol} / \mathrm{L})$.

Based on the guidelines established for the diagnosis of PNS, the patient's clinical presentation appeared consistent with definite PNS. The classification of PNS as definite requires a non-classical syndrome with onconeural antibodies (well-characterized or not) and cancer that develops within five years of the diagnosis of the neurological disorder. The patient's symptoms of dysphagia were felt to be a non-classical manifestation of PNS, which was further supported by the identification of the onconeural antibody for VGCC and a negative workup for other possible etiologies.

The patient declined any further treatment. She was made comfortable as per her wishes and the family members. The patient succumbed to her disease shortly.

\section{Discussion}

We are presenting a case of a patient with oropharyngeal dysphagia as a paraneoplastic neurologic complication of breast cancer. Our case report confirms the importance of considering PNS when investigating breast cancer patients presenting with neurological symptoms of unknown etiology. In most cases of PNS, neurological symptom onset precedes a tumor diagnosis [15]. However, there have been reported cases of patients developing PNS despite treatment of primary breast cancer [16]. Other case reports showed improvement of the neurological symptoms after therapy of the primary tumor [17].

The presence of VGCC antibodies has been frequently found in association with Lambert-Eaton syndrome and SCLC. However, there are reported cases of VGCC antibodies with breast cancer that lead to various phenotypes ranging from muscle weakness, chorea, dysphagia, dysarthria, and even respiratory failure $[18,19]$.

Given the rare incidence of PNS, treatment strategies have been based on limited case series and cohort studies. Treatment of PNS can be differentiated into the treatment of the underlying tumor and immunotherapy. Prompt initiation of tumor therapy should be instituted according to current clinical practice guidelines. Treatment of the underlying tumor enables stabilization or improvement of the neurological disorders [20].

There have been no reported cases of PNS in breast cancer patients improving with immunotherapy. However, immunosuppressive therapy has been used with varying degrees of success. Intravenous immunoglobulin (IVIG), steroids, and plasma exchange were more successful in the management of PNS in breast cancer patients. Other immunosuppressive agents used include cyclophosphamide, mycophenolate, and rituximab [1].

\section{Conclusions}

PNS is a rare neurological complication in breast cancer, which makes developing guidelines for the diagnosis and treatment of these disorders challenging. More prospective studies and clinical trials are required to draw more evidence-based conclusions. However, PNS should be considered in the differential of patients presenting with unexplained neurological symptoms. Since diagnosing PNS can take time, we recommend the prompt initiation of treatment in cases with a high index of suspicion since it may help stabilize or improve the neurological disorder.

\section{Additional Information}

\section{Disclosures}

Human subjects: Consent was obtained or waived by all participants in this study. Conflicts of interest: In compliance with the ICMJE uniform disclosure form, all authors declare the following: Payment/services info: All authors have declared that no financial support was received from any organization for the submitted work. Financial relationships: All authors have declared that they have no financial relationships at present or within the previous three years with any organizations that might have an interest in the submitted work. Other relationships: All authors have declared that there are no other relationships or activities that could appear to have influenced the submitted work.

\section{References}

1. Fanous I, Dillon P: Paraneoplastic neurological complications of breast cancer . Exp Hematol Oncol. 2015, 
5:29. 10.1186/s40164-016-0058-x

2. Graus F, Delattre JY, Antoine JC, et al.: Recommended diagnostic criteria for paraneoplastic neurological syndromes. J Neurol Neurosurg Psychiatry. 2004, 75:1135-40. 10.1136/jnnp.2003.034447

3. Sabater L, Hoftberger R, Boronat A, Saiz A, Dalmau J, Graus F: Antibody repertoire in paraneoplastic cerebellar degeneration and small cell lung cancer. PLoS One. 2013, 8:60438. 10.1371/journal.pone.0060438

4. Peterson K, Rosenblum MK, Kotanides H, Posner JB: Paraneoplastic cerebellar degeneration. I. A clinical analysis of 55 anti-Yo antibody-positive patients. Neurology. 1992, 42:1931-7. 10.1212/wnl.42.10.1931

5. Shams'ili S, Grefkens J, de Leeuw B, et al.: Paraneoplastic cerebellar degeneration associated with antineuronal antibodies: analysis of 50 patients. Brain. 2003, 126:1409-18. 10.1093/brain/awg133

6. Tiryaki E, Horak HA: ALS and other motor neuron diseases. Continuum (Minneap Minn). 2014, 20:1185-207. 10.1212/01.CON.0000455886.14298.a4

7. Ahmed A, Simmons Z: Pseudobulbar affect: prevalence and management. Ther Clin Risk Manag. 2013, 9:483-9. 10.2147/TCRM.S53906

8. Titulaer MJ, Lang B, Verschuuren JJ: Lambert-Eaton myasthenic syndrome: from clinical characteristics to therapeutic strategies. Lancet Neurol. 2011, 10:1098-107. 10.1016/S1474-4422(11)70245-9

9. Graus F, Lang B, Pozo-Rosich P, Saiz A, Casamitjana R, Vincent A: P/Q type calcium-channel antibodies in paraneoplastic cerebellar degeneration with lung cancer. Neurology. 2002, 59:764-6. 10.1212/wnl.59.5.764

10. Fernandez-Torron R, Arcocha J, Lopez-Picazo JM, et al.: Isolated dysphagia due to paraneoplastic myasthenic syndrome with anti-P/Q-type voltage-gated calcium-channel and anti-acetylcholine receptor antibodies. Neuromuscul Disord. 2011, 21:126-8. 10.1016/j.nmd.2010.10.003

11. Kornitzer J, Taha F, Segal E: Clinical presentation and outcome in autoimmune encephalitis associated with N-type voltage-gated calcium channels in children. J Child Neurol. 2019, 34:499-505. 10.1177/0883073819840448

12. Frey J, Murray A: Autoimmune encephalitis with elevated N-type calcium channel antibodies as a multiple sclerosis mimic. Mult Scler Relat Disord. 2018, 26:201-3. 10.1016/j.msard.2018.09.028

13. Younes K, Lepow LA, Estrada C, Schulz PE: Auto-antibodies against P/Q- and N-type voltage-dependent calcium channels mimicking frontotemporal dementia. SAGE Open Med Case Rep. 2018, 6:2050313X17750928. 10.1177/2050313X17750928

14. Venkatraman A, Opal P: Paraneoplastic cerebellar degeneration with anti-Yo antibodies - a review . Ann Clin Transl Neurol. 2016, 3:655-63. 10.1002/acn3.328

15. Gatti G, Simsek S, Kurne A, et al.: Paraneoplastic neurological disorders in breast cancer. Breast. 2003, 12:203-7. 10.1016/s0960-9776(03)00011-0

16. Barata PC, Morgado J, Sousa AP, et al.: Breast cancer presents with a paraneoplastic neurologic syndrome . Case Rep Oncol. 2012, 5:616-21. 10.1159/000345692

17. Kawasoe T, Yamamoto Y, Okumura Y, Iwase H: A case report of paraneoplastic neurological syndrome associated with occult breast cancer. Breast Cancer. 2006, 13:202-4. 10.2325/jbcs.13.202

18. Pera E, Kaemmerer E, Milevskiy MJG, et al.: The voltage gated $\mathrm{Ca}(2+)$-channel Cav3.2 and therapeutic responses in breast cancer. Cancer Cell Int. 2016, 16:24. 10.1186/s12935-016-0299-0

19. Chang K, Lwanga A, Kaur T, Helgason C: P/Q and N-type voltage-gated calcium channel binding antibodies associated with paraneoplastic chorea and mixed invasive ductal and lobular carcinoma of the breasts in an elderly patient. Cureus. 2018, 10:e3097. 10.7759/cureus.3097

20. Wood JP, Haynes AP, Cheung KL: A paraneoplastic manifestation of metastatic breast cancer responding to endocrine therapy: a case report. World J Surg Oncol. 2008, 6:132. 10.1186/1477-7819-6-132 